controls, one which adjusts the gain and the other which introduces a bias to adjust the zero value of intensity (see paper no. 2). In spanning the gap between what may be observed visually and what may be recorded photographically on planets, these contrast adjustments have proved to be of considerable value.

The first experiments suggested techniques which should improve future tests. One procedure would be to place a shutter having speeds up to I/200 sec. in front of the photocathode and to synchronize it with a 'suppressor circuit' which keeps the kinescope screen dark except during the frame scan immediately following the exposure of the cathode. The camera used to photograph the kinescope screen can then operate continuously with an open shutter.

With an image-tube system having a larger number of frame scans, a more suitable spectral response, and a synchronized shutter, it should be possible to overcome present limitations and to surpass any planet photographs thus far obtained.

\title{
6. SOME APPLICATIONS OF IMAGE CONVERTERS IN THE U.S.S.R.
}

By V. I. Krassovsky, Geophysical Institute of the Academy of Sciences, U.S.S.R.

In order to photograph astronomical objects of extremely small intensity or to obtain their spectra, astronomers are obliged to use not only large telescopes and photographic materials of the highest sensitivity, but also extremely long exposures sometimes lasting many hours. If the application of image converters is aimed at detecting undiscovered objects of extremely low intensity, it is also necessary that converters should be capable of long exposures. An increase in sensitivity, without provision for prolonging exposures, may only shorten the exposure times for already known faint objects or their spectra. For astronomical investigations it is also highly desirable to extend the spectral sensitivity, particularly into the infra-red.

Various photo-electric methods were introduced into astronomical practice during the past decade. Their principles, their main characteristics, and the properties of photocathodes are sufficiently described in the literature. It might, therefore, be advisable to recall here certain of their peculiarities which determine their role in astronomical investigations.

As is known, the slow fall of sensitivity in the long wave-lengths in the case of cesiumoxide-on-silver photocathodes is extremely valuable. It does not exceed an order of magnitude between the visible region and about $I \mu$, whereas the sensitivity of the best photographic emulsions decreases several orders of magnitude in that range.

The possibility of a considerable reduction in size by means of electron-optical demagnification inside an image converter permits shortening exposures considerably. This is equivalent to an increase in the focal ratio, which cannot be realized by means of ordinary optical methods(r). This method is advisable in a number of cases, in spite of the resulting diminution in the distinctness of images.

Artificial cooling of the photocathode (for example, by means of solid carbon dioxide) decreases the dark current to such an extent that exposures of several tens of hours become possible(r). The generation and use of high accelerating voltages of $15-20 \mathrm{kV}$. is quite feasible. The production of fine-structure fluorescent screens of good quality, the emission of which is matched to the spectral response of photographic emulsions, meets no insurmountable difficulties.

However, all photo-electric image-converter systems possess a number of essential deficiencies. The most important restriction on the application of image converters is the small size of the working field when flat cathodes are used. The reception of stationary images is hampered when photo-electric image converters of long dimensions are used; the shift of images is caused in the first place by the external magnetic field, which is particularly evident when a parallactic arrangement of the instrument is used. Ordinary photo-electric image converters permit resolving on the photocathode about $20-40$ line 
pairs within a field $\mathrm{I} \mathrm{cm}$. in diameter, but such resolving power cannot be realized in practice with long exposures. It is, therefore, most advisable to use short photo-electric systems, which do not, however, permit a working field of large dimensions. It is extremely difficult to obtain strictly constant sensitivity over the whole surface of the photocathode, which makes the photometric reduction of the resulting photographs rather complicated.

Let us also make some remarks regarding individual properties of various systems of photo-electric photography.

Particularly fine is the system of photography in which the direct action of accelerated photo-electrons upon a photographic emulsion is made use of in the manner described by Prof. Lallemand. This method does not, however, permit long exposures, the importance of which was emphasized above. This is the result of the fact that the sensitivity of the photo-electric cathode is rapidly decreasing due to the desorption of gases from the photographic emulsion. The gain as compared with conventional unaided photography is appreciable only for extremely short exposures. The incorporation of a Leonard window in the anode of a photo-electric tube ensures an isolated outer disposition of the photographic emulsion, but secure Leonard windows of large dimensions have not yet been realized in practice.

New photo-electric image converters having internal secondary photo-electric amplification are described in the literature; also described are converters consisting of several stages connected in series with direct contact between each fluorescent screen and the photocathode of the succeeding stage. The images obtained with such systems, however, are not sufficiently distinct. An increase in the length of converter systems is followed by a decrease in the stability of the image position. As the author has already pointed out, it is extremely important to obtain photographic images with separate scintillations created by individual photo-electrons (6). This was not yet, however, realized in practice except under laboratory conditions when long exposures were used.

The most simple system is an ordinary photo-electric image converter whose fluorescent screen is photographed with the aid of properly corrected lenses of medium focal ratios. Unfortunately, only one-tenth of the total light flux emitted by the fluorescent screen can at best be utilized in this case. The use of liquid-immersion objective lenses of the highest possible focal ratios permits this deficiency to be reduced, but it is then impossible to obtain an image larger than about $\mathrm{I} \mathrm{cm}$. diameter.

An ordinary photo-electric image converter is most effective when the internal side of a mica window as thin as 5-20 $\mu$ is covered by a fluorescent layer. By placing the photographic film outside such a mica window and creating a vacuum between them, one can obtain a tight elastic optical contact over a field I cm. in diameter. Our photos of the infra-red spectrum of the night $\operatorname{sky}(7,8,9)$ were obtained in this way with the shortest possible exposures.

In our photos obtained in the infra-red in the spring of 1947 , R Leonis was a bright star; it is relatively faint in the visual region of the spectrum(5).

Photo-electric image converters were applied at the Crimean Astrophysical Observatory of the Academy of Sciences of the U.S.S.R. for programme photography of astronomical objects and of the infra-red night air-glow. By means of an $f / 2$ prism spectrograph and an ordinary photo-electric image-converter tube we were able in 1948 and r949 to photograph infra-red spectra of the night air-glow with a dispersion of $\mathrm{I} 200-2400 \AA$ per mm. $(\mathrm{I}, 2,3)$. In the region up to $8800 \AA$ were reproduced the bands discovered by Slipher in 1933 . In r950 Meinel, on more distinct photographs of the infra-red spectra up to $8800 \AA$, identified them as hydroxyl bands. But long before that identification we discovered infra-red bands in the night air-glow in the region from $8800 \AA$ to II,000 $\AA$. The author, together with A. A. Kaliniak and V. B. Nikonov (4, 5), used short-focus cameras on the same spectrograph in 1948 and I949 to obtain $20-40 \mathrm{~min}$. photographs in the infra-red near I0,000 $\AA$ of an area around the centre of the Galaxy. Together with V. T. Lukashenja $(7,8,9)$, the author used a diffraction spectrograph in I950 and I95I to obtain contact photographs of the infra-red spectrum of the night air-glow in the vicinity of 
I0,000 $\AA$. In this case the dispersion was $150-200 \AA / \mathrm{mm}$. for $f / 2$. A. B. Severny (Ix) and G. S. Ivanov-Kholodny(r2) successfully photographed solar prominences and their infra-red spectra.

A. A. Kaliniak ( $\left(_{3}\right)$ obtained infra-red photographs of the solar corona by means of a photo-electric image converter attached to the coronagraph of the Mountain Station of the Central Astronomical Observatory of the Academy of Sciences of the U.S.S.R. He also obtained similar photographs during the solar eclipse of 1954.

At the Geophysical Institute of the Academy of Sciences of the U.S.S.R., B. A. Bagariatzky and M. I. Mordukhovitch (ro) have successfully photographed the spectra of aurorae up to II,000 $\AA$ by means of the prism spectrograph used earlier at the Crimean Astrophysical Observatory. Using an ordinary photo-electric image converter attached to an $f / 2$ diffraction spectrograph with a dispersion of $250 \AA / \mathrm{mm}$., B. A. Bagariatzky and N. I. Fedorova have obtained more detailed photographs of the infra-red spectra of aurorae and the night air-glow up to $I I, 000 \AA$. In the latter case the exposures reached 5-Io hr.

By means of a photo-electric image converter attached to an $f / \mathrm{I} \cdot 4$ camera of $90 \mathrm{~cm}$. focus, P. V. Scheglov (Sternberg Astronomical Institute) obtained at the Crimean Astrophysical Observatory a number of direct photographs of stars in the infra-red.

\section{REFERENCES}

(I) V. I. Krassovsky, C.R. Acad. Sci. U.R.S.S. 66, no. I, 1949.

(2) V. I. Krassovsky, C.R. Acad. Sci. U.R.S.S. 70, no. 6, 1950.

(3) V. I. Krassovsky, Publ. Crim. Obs. 5, roo, 1950.

(4) A. A. Kaliniak, V. I. Krassovsky, and V. B. Nikonov, C.R. Acad. Sci. U.R.S.S. 66, no. I, 1949.

(5) A. A. Kaliniak, V. I. Krassovsky, and V. B. Nikonov, Publ. Crim. Obs. 6, I 19, I950.

(6) V. I. Krassovsky, C.R. Acad. Sci. U.R.S.S. 78, no. 3, r95I.

(7) V. T. Lukashenja and V. I. Krassovsky, C.R. Acad. Sci.U.R.S.S. 79, no. 2, $195 \mathrm{I}$.

(8) V. I. Krassovsky and V. T. Lukashenja, C.R. Acad. Sci. U.R.S.S. 8o, no. 5, I95 I.

(9) V. T. Lukashenja and V. I. Krassovsky, C.R. Acad. Sci. U.R.S.S. 81, no. 5, 195 I.

(Io) B. A. Bagariatzky and M. I. Mordukhovitch, C.R. Acad. Sci. U.R.S.S. 82, no. I, I952.

(I I) A. B. Severny, C.R. Acad. Sci. U.R.S.S. 74, no. 2, 1950.

(12) G. S. Ivanov-Kholodny, Publ. Crim. Obs. 8, Ir5, I95 r.

(13) A. A. Kaliniak, C.R. Acad. Sci. U.R.S.S. 72, 66r, r95o.

\section{COMPARISON OF IMAGE-TUBE SYSTEMS}

\section{By A. D. CODE and R. H. HARDIE, Washburn Observatory and Lowell Observatory}

The high quantum efficiency of the cathode in a photo-electric image device results in a greater number of statistically significant photo-events per unit time than can be obtained with an unaided photographic plate. The maximum information is contained in the initial photo-electron flux at the photocathode. The measurement or storage of these primary events represents the major problem in image-tube design.

A figure of merit or a detectability criterion for an image surface is derived on the basis of signal-to-noise ratio considerations. The performance of any specific image device is to be compared with this 'ideal' performance. The principal factors responsible for 'non-ideal' performance, namely, saturation and subsequent circuit noise, are examined. An expression for the sky-saturated case is derived which is equivalent to that presented by W. A. Baum in paper no. 3 .

It is suggested that the use of fine-grain plates, image magnification, or multiple exposures are practical methods of greatly extending the saturation limit. By these 